\title{
Traumatic Retrobulbar Haemorrhage: Aetio-pathology and management
}

\author{
Gagan Sabharwal, ${ }^{1}$ Amit Agrawal, ${ }^{2}$ Shikha Baisakhiya ${ }^{3}$ \\ ${ }^{1}$ Department of Oral and Maxillofacial Surgery, AB Shetty Memorial Institute of Dental Sciences, Mangalore \\ ${ }^{2}$ Department of Neurosurgery, Datta Meghe Institute of Medical Sciences, Sawangi (Meghe), Wardha \\ ${ }^{3}$ Department of Ophthalmology, Datta, Meghe Institute of Medical Sciences, Sawangi (Meghe) Wardha
}

\section{SUMMARY}

Retrobulbar haematoma following blunt orbital trauma is a rare, but potentially serious complication, since it can evolve rapidly from visual impairment to permanent loss of vision. This sight-threatening situation most commonly arises from orbital bleeding accompanying undisplaced fractures of the orbital walls, an event that increases the pressure inside the orbit and results in vascular damage to the optic nerve. The clinical presentation includes pain, exophthalmos with proptosis, and internal ophthalmoplegia, with impairment or loss of the pupillary reflex. A thin-layer orbital CT scan is an essential diagnostic aid. Therapy is based on orbital decompression, via different surgical approaches, with the intention of reducing the pressure on the nerve and vascular structures inside the orbit. Emergent management is of utmost importance as any delay between the onset of symptoms and treatment can have a significant effect on recovery.

Key words: orbital trauma, retrobulbar haemorrhage, retrobulbar haematoma, visual loss, orbital fracture, mid-face fractures, facial trauma

\section{INTRODUCTION}

Retrobulbar haematoma following blunt orbital trauma is a rare, but potentially serious complication, since it can evolve rapidly from visual impairment to permanent loss of vision. if the condition is not diagnosed and treated early. ${ }^{1}$ Oral and maxillofacial surgeons are regularly referred patients with injuries around the orbit, and as a result, we should ensure that all members of our team recognize this medical condition immediately and manage it with minimal morbidity. ${ }^{2,3}$ The aetiology, pathogenesis and management of patients with retrobulbar haemorrhage are discussed in this paper.

\section{Aetiology}

The causes of retrobulbar haemorrhage include surgical reduction of orbital fractures and mid-face fractures, cosmetic or reconstructive operations on the eyelid, endoscopic operations on the sinus, retrobulbar injections, direct consequence of blunt trauma with or without orbital fracture and thrombolytic agents. ${ }^{3-12}$ Loss of vision as a complication of maxillofacial trauma occurs in $3-12 \%$ of the cases seen and diagnosed. ${ }^{4}$ The incidence of retrobulbar haemorrhage with visual loss from fractures of the zygomatic complex is low $(0.3 \%){ }^{5}$ It is less frequently reported as a direct consequence of blunt trauma with or without orbital fracture. ${ }^{10,11,12}$ Local anaesthesia in the retrobulbar space for surgical procedures is a time-honoured practice that provides akinesia and analgesia to the eye. ${ }^{13-18}$ This procedure, however, rarely becomes complicated by the development of a retrobulbar orbital hemorrhage (incidence0.75 per cent) with potential risk to the vision. ${ }^{15} \mathrm{~A}$ retrobulbar haematoma can result from an injury to one of the blood vessels in the highly vascular orbital fat, or to one of the extraocular muscles with a variable degree of proptosis. ${ }^{15,19}$ Occasionally, thrombolytic agents, such as tissue plasminogen activator (TPA), commonly employed early in the management of patients with acute myocardial infarction can result in lysis of a haemostatic plug in an arterial vessel within the orbit that was punctured during the delivery of the anaesthetic. ${ }^{20}$

\section{PATHOGENESIS}

This sight-threatening situation most commonly arises from orbital bleeding accompanying undisplaced fractures of the orbital walls. This increases the pressure inside the orbit and results in vascular damage to the optic nerve. ${ }^{1,10}$ Owing to the anatomy of the orbit, which has inflexible walls, even small haematomas that might be insignificant sometimes could have catastrophic consequences. In fact, the orbital

*Correspondence: Dr Amit Agrawal, Division of Neurosurgery, Datta Meghe Institute of Medical Sciences, Sawangi (Meghe) Wardha, India. Email: dramitagrawal@gmail.com,dramit_in@yahoo.com 
tissues seem to respond to fluid overload in such a way that once the hydraulic system of the orbit is full, even small changes in volume can cause a dramatic increase in orbital pressure..$^{21}$ An orbital haematoma can cause complications ranging from vascular compression to acute stretching of the optic nerve resulting from exophthalmos. The accompanying retrobulbar oedema may reduce retinal perfusion and compress the long and short ciliary vessels, leading to ischaemic damage to the optic nerve. ${ }^{10,11}$

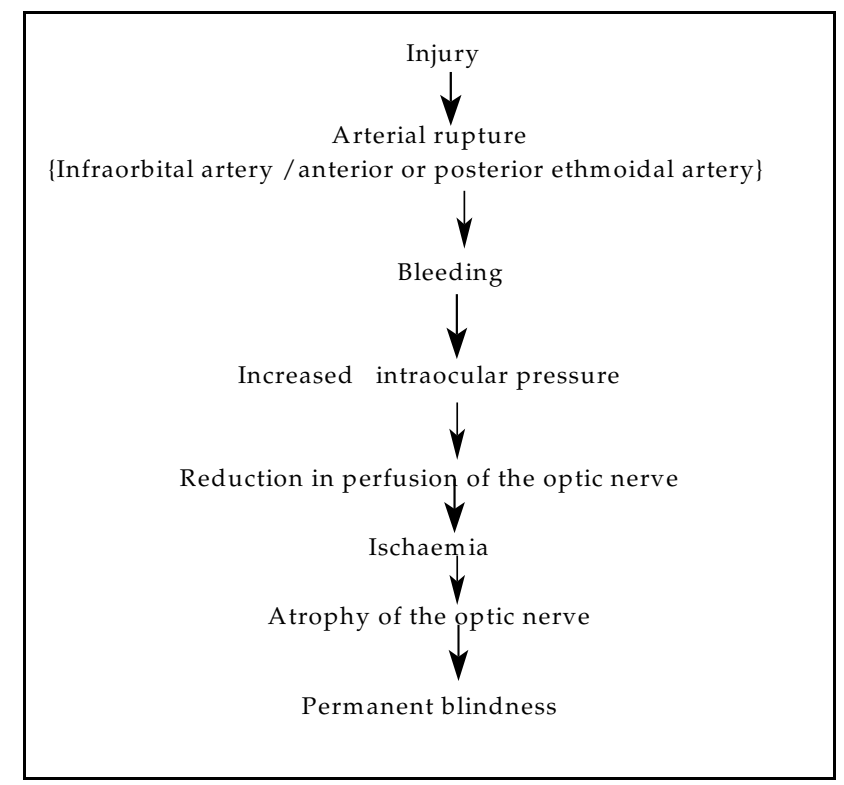

Figure1. Mechanism of retrobulbar haematoma

\section{Clinical Features}

A thorough ophthalmological examination should be made in all patients with orbital injuries. ${ }^{22}$ A patient presenting with three or more of the following signs and symptoms probably has a retrobulbar haematoma: pain, proptosis, chemosis, diplopia, subconjunctival haemorrhage, increased intraocular pressure, tense globe, decreasing visual acuity, loss of direct pupillary light reflex, and ophthalmoplegia. ${ }^{12}$ The appearance and size of the haematoma can be assessed on a four-grade scale, e.g., 1 (spot ecchymosis), 2 (lid ecchymosis involving half the lid surface area or less), 3 (lid ecchymosis all round the eye, no increase in intraocular pressure), and 4 (retrobulbar haemorrhage with increased intraocular pressure). ${ }^{23}$

\section{MANAGEMENT}

In animal models, it has been shown that ischaemia, due to transient central retinal artery occlusion, is well tolerated up to 100 minutes; however, irreparable damage can occur after ischaemia of 105 minutes. ${ }^{24}$ Therefore, early diagnosis and aggressive management of retrobulbar haemorrhage is essential as vision may be preserved if intervention is prompt. $^{25}$

\section{Medical management}

Pharmacological management is just as important as surgery and has even been shown to produce a satisfactory outcome when used as sole treatment. ${ }^{6}$ It can be instituted immediately after the diagnosis or while lateral canthotomy is being done. Hyperosmotic agents, carbonic anhydrase inhibitors, and corticosteroids are the mainstay of treatment. Osmotic agents, such as mannitol, shrink the vitreous and reduce the volume of tissue in the orbit. Acetazolamide reduces intraocular pressure by inhibiting carbonic anhydrase and dramatically reducing the production of aqueous. If there is an improvement in the vision and reduction in the local signs than conservative treatment should be continued for 5-7 days. ${ }^{26}$ Other simple conservative measures that can be overlooked include elevation of the head, topical ice packs, and avoidance of dressings that can often be compressive and increase intraocular pressure. ${ }^{5}$

\section{Surgical management}

Knowledge of orbital anatomy is important to enable the surgeon to do the lateral canthotomy. The inferior crus and superior crus of the lateral canthal tendon form a common tendon at the point of attachment to the inner part of the lateral orbital rim (Whitnall's tubercle). It is the inferior crus of the lateral canthal tendon that is cut in lateral canthotomy. ${ }^{27}$ Drainage of the haematoma and bipolar coagulation of bleeding vessels can be performed via subperiosteal dissection of the orbit, with the incision of the periosteum over the haematoma and a blunt dissection of the orbital retrobulbar structures. Access can be obtained through a subtarsal lower eyelid incision, or an upper eyelid or coronal approach. A temporary marginotomy was occasionally required to gain adequate surgical visibility of the retrobulbar area. ${ }^{1}$ The author has described a simplified technique of orbital decompression that involves fracturing the orbital floor and inserting a clamp in the orbit through a small trans-conjunctival incision. ${ }^{28}$

\section{PREVENTION}

The prevention of the loss of vision in patients with retrobulbar haemorrhage is of utmost importance as most of the episodes have been reported to occur overnight. It has been recommended that the patients should be monitored every 15 minutes for first 2 hours, every 30 minutes for second 2 hours and hourly for the next 16 hours or overnight. ${ }^{2,29}$ 


\section{CONCLUSION}

A review of the related literature confirms that although retrobulbar haemorrhage is a rare complication of blunt periorbital trauma, irreversible visual sequelae can be prevented by prompt diagnosis and immediate surgical and pharmacological therapy. ${ }^{12}$ Visual acuity must be monitored carefully over time in all facial trauma patients, and an accurate CT scan assessment should be performed in any suspected case. Early diagnosis and immediate treatment based on surgical decompression are essential to prevent permanent damage. ${ }^{1}$

\section{REFERENCES}

1. Gerbino G, Ramieri GA, Nasi A. Diagnosis and treatment of retrobulbar haematomas following blunt orbital trauma: A description of eight cases. Int J Oral Maxillofac Surg 2005; 34(2): 127-31.

2. Bater MC, Ramchandani PL, Brennan PA. Post-traumatic eye observations. Br J Oral Maxillofac Surg 2005; 43: 410-416.

3. Girotto JA, Gamble WB, Robertson B, Muehlberger T, Mayer M, Zinreich J, Ilif N, Miller N, Manson PN. Blindness after reduction of facial fractures. Plast Reconstr Surg 1998; 102: 1821-1834.

4. Ioannides C, Freihofer HPM, Bruaset I. Trauma of the upper third of the face. J Maxillofac Surg 1984; 12: 255-261.

5. Ord RA. Postoperative retrobulbar haemorrhage and blindness complicating trauma surgery. Br J Oral Surg 1981; 19: 202-7.

6. DeMere M, Wood T, Austin W. Eye complications with blepharoplasty or other eyelid surgery. Plast Reconstr Surg 1974; 53: 364-370.

7. Saussez S, Choufani G, Brutus JP,Cordonnier M, Hassid S. Lateral canthotomy: A simple and safe procedure for orbital haemorrhage secondary to endoscopic sinus surgery. Rhinology 1998; 36: 37-39.

8. Cionni RJ, Osher RH. Retrobulbar haemorrhage. Ophthalmology 1991; 98: 153-1155.

9. Rubens $\mathrm{S}$. The incidence of complications associated with retrobulbar injection of anaesthetic for ophthalmic surgery. Acta Ophthalmol Copenh 1992; 70: 836-838.

10. Bailey WK, Paul C, Evans LS. Diagnosis and treatment of retrobulbar haemorrhage. J Oral Maxillofac Surg 1993; 51: 780-781.

11. Korinth MC, Ince A, Banghard W, Huffmann BC, Gilsbach JM. Pterional orbital decompression in orbital haemorrhage and trauma. J Trauma 2002; 53: 73-78.

12. Rosdeutscher JD, Stadelmann WK. Diagnosis and treatment of retrobulbar haematoma resulting from blunt periorbital trauma. Ann Plast Surg 1998; 41: 618-62.
13. Goldsmith MO. Occlusion of the central retinal artery following retrobulbar haemorrhage. Ophthalmologica 1967; 153: $191-6$.

14. Katz B, Herschler J, Brick DC. Orbital haemorrhage and prolonged blindness: a treatable posterior optic neuropathy. Br J Ophthalmol 1983; 67: 549-53.

15. Kaushik NC. Orbital haemorrhage following retrobulbar injection. Indian J Ophthalmol 1988; 36: 128-30.

16. Klein ML, Jampol CM, Condon PI, Rice TA, Serjeant GR. Central retinal artery occlusion without retrobulbar hemorrhage after retrobulbar anesthesia. Am J Ophthalmol 1982; 93: 573-7.

17. Krohel GB, Wright JE. Orbital hemorrhage. Am J Ophthalmol 1979; 88: 254-8.

18. Subbiah S, McGimpsey S, Best RM. Retrobulbar hemorrhage after sub-Tenon's anesthesia. J Cataract Refract Surg 2007; 33(9): 1651-2.

19. Weiss IS. Retrobulbar haemorrhage occurring after placement of superior rectus suture. Am J Ophthalmol 1975; 80: 153-4.

20. Chorich LJ, Derick RJ, Chambers RB, et al. Hemorrhagic ocular complications associated with the use of systemic thrombolytic agents. Ophthalmology 1998; 105: 428-31.

21. Rootman J, Stewart B, Goldberg RL. Orbital Surgery: A conceptual approach. Philadelphia: Lippincott-Raven 1995.

22. Dutton GN, Al-Qurainy IA, Stassen LFA, Titherington DM, Moos KF, El-Attar A. Ophthalmic consequences of mid-face trauma. Eye 1992; 6: 86-9.

23. Kallio H, Paloheimo M, Maunuksela EL. Haemorrhage and risk factors associated with retrobulbar/peribulbar block: a prospective study in 1383 patients Br J Anaesth 2000; 85: 70811.

24. Hayreh SS, Kolder HE, Weingeist TA. Central retinal occlusion and retinal tolerance time. Ophthalmology 1980; 87: 75-8.

25. Heinze JB, Hueston JT. Blindness after blepharoplasty: Mechanism and early reversal. Plast Reconstr Surg 1978; 61: 347-54.

26. Wood CM. The medical management of retrobulbar haemorrhage complicating facial fractures: a case report. $\mathrm{Br} J$ Oral Maxillofac Surg 1989; 27: 291-5.

27. Goodall KL, Brahma A, Bates A, Leatherbarrow B. Lateral canthotomy and inferior cantholysis: an effective method of urgent orbital decompression for sight threatening acute retrobulbar haemorrhage. Injury 1999; 30: 485-90.

28. Liu D. A simplified technique of orbital decompression for severe retrobulbar haemorrhage. Am J Ophthalmol 1993; 116: 34-37.

29. Hislop WS, Dutton GN. Retrobulbar haemorrhage: Can blindness be prevented? Injury 1994; 25: 663-5. 